\title{
Evaluation and Estimation of Fatigue on Long-distance Driving
}

\author{
Ryosuke Sugie $^{\mathrm{a}, *}$, Toshiya Arakawa ${ }^{\mathrm{b}}$, Kazuhiro Kozuka $^{\mathrm{b}}$ \\ ${ }^{a}$ Aichi University of Technology, Graduate School of Engineering \\ 50-2, Manori, Nishihasama-cho, Gamagori, Aichi, 443-0047 Japan \\ ${ }^{\mathrm{b}}$ Aichi University of Technology, 50-2, Manori, Nishihasama-cho, Gamagori, Aichi, 443-0047 Japan \\ *Corresponding Author: arakawa-toshiya@aut.ac.jp
}

\begin{abstract}
Traffic accidents caused by fatigue and/or overwork driving on driving for ling-time never ceased and we are attempting to evaluate and estimate fatigue. 3 participants drove on a highway course consisted of a slow lane and a passing lane for about an hour and were measured their R-R interval. Their fatigue level was analyzed based on LF/HF, which is calculated by Fast-Fourier-Transformed (FFT) R-R interval. From experiment and data analysis, 2 participants were seemed to get to be fatigue state judging from increasing of $\mathrm{LF} / \mathrm{HF}$.
\end{abstract}

Keywords: Fatigue, Heartbeat, LF/HF, Evaluation, Estimation.

\section{Introduction}

4,000 km highway network in Japan has been opened and extended from 1989 to 2013 , and it means that it is suitable for drivers to drive for long-time(1).

The number of fatal traffic accidents caused by fatigue and/or overwork driving tends to decrease because of legal effort and business effort. However, There are cases that fatigue and/or overwork caused a big fatal traffic accident, which was happened on April 2012 and March 2013(2)(3). Thus, traffic accident caused by fatigue and/or overwork driving on driving for ling-time never ceased and we are attempting to evaluate and estimate fatigue.

It is common physiological method to evaluate fatigue by heartbeat, breathing, perspiration(4) and brainwave(5). Nowadays a fatigue detection system by flicker test is developing based on the correlation the low flicker value and happening incident(6). We are attempting to evaluate and estimate fatigue based on heartbeat from the point of view the cost of system and the probability of real-time detection and evaluation.

\section{Experiment}

3 participants (Participant A, B and C) were joined to experiment in order to evaluate the heartbeat on driving long-time. 2 participants were 21 years old, and 1 participant was 19 years old.

All participants were taught to wear the wearable-typed heart rate monitor (WHS-1, made by Union Tool, Co.) and measured their pulse wave and R-pressure pulse interval (also known as the R-R interval). They were also taught to drive using driving simulator (D3Sim, made by Mitsubishi Precision Co, LTD.)

Firstly, all participants stayed calm with clothing their eyes and their R-R interval were measured. Secondly, they drove on a simple urban course about 5 minutes and were accustomed to driving before experiment as training because all participants were not used to drive using driving simulator. After that, all participants drove on a highway course consisted of a slow lane and a passing lane for about an hour. Some obstacles were set and all participants were urged to avoid these obstacles on driving so as not to get to be drowsy state because of monotonous driving. In addition that, "Jikaku-sho Shirabe (questionnaire of subjective fatigue)" and "Hirou-bui Shirabe (questionnaire of fatigue part)," which is adopted by a society for the study of work fatigue of Japan Society for Occupational Health, were implemented after and before the experiment. Subjective fatigues of all participants were surveyed by these questionnaires. "Jikaku-sho Shirabe" can evaluate the subjective fatigues as five categories such as sleepiness, nervous, discomfort, sluggish and blurred. We obtained informed consent before experiment from all participants. 


\section{Results}

\subsection{Experimental Results}

We analyzed LF/HF which is obtained from the power spectrum by Fast-Fourier-Transformed (FFT) R-R interval. Here, LF means intermediate frequency component of power spectrum and HF means high frequency component of power spectrum. In this paper, LF is defined as a range of power spectrum from $0.05 \mathrm{~Hz}$ to $0.15 \mathrm{~Hz}$ and $\mathrm{HF}$ is defined as a range of power spectrum from $0.15 \mathrm{~Hz}$ to 0.40 $\mathrm{Hz}$. The ratio of $\mathrm{LF}$ to $\mathrm{HF}, \mathrm{LF} / \mathrm{HF}$, is said to be useful indicator of tension or fatigue.

It is usual method that the frequency is analyzed by AR model, which is efficient to analysis a short time data because of high spatial solution. However, in this paper, we analysis a long time data and it is said that there is a similar tendency between the frequency analysis result by AR model and by FFT(7). Thus, we analyzed R-R interval data and LF/HF data by FFT. LF/HF was $\mathrm{z}$ transformed because $\mathrm{LF} / \mathrm{HF}$ is different between each participant.

Firstly, we confirmed whether participants felt fatigued or not from "Jikaku-sho Shirabe" and "Hirou-bui Shirabe." From the answer of "Jikaku-sho Shirabe", score of some categories, such as "can't think clearly", "yawning", "want to lie down", "get bleary", and so on, of after experiment was higher than that of before experiment. From the answer of "Hirou-bui Shirabe", score of some categories, such as "neck", "left elbow and forearm", "right upper arm", "left buttock and thigh", "right elbow and forearm", and so on, of after experiment was higher than that of before experiment. Thus, it is found that all participants felt fatigued from questionnaire.

We compared average $\mathrm{LF} / \mathrm{HF}$ value when participants were closing their eyes calmly and when they were driving using driving simulator. The result shows Fig. 1 and Table 1. From Fig. 1 and Table 1, LF/HF value, which wasn't $z$ transformed, during calm with closing participants' eyes tends to be smaller than that on driving using driving simulator. It is found that the mental stress on driving tends to be high. Next, the ratio of z-transformed LF/HF value of 15 minutes (900 seconds) after the start of driving and that of 5 minutes ( 300 seconds) each after 15 minutes after the start of driving was calculated. The result is shown in Fig. 2 and Table 2. Here, from driver model of Arakawa et al.(8), drivers tend to be in "excessive state" just after the start of driving, so $\mathrm{LF} / \mathrm{HF}$ value tends to be high. Thus, the data before 15 minutes after the start of driving was omitted

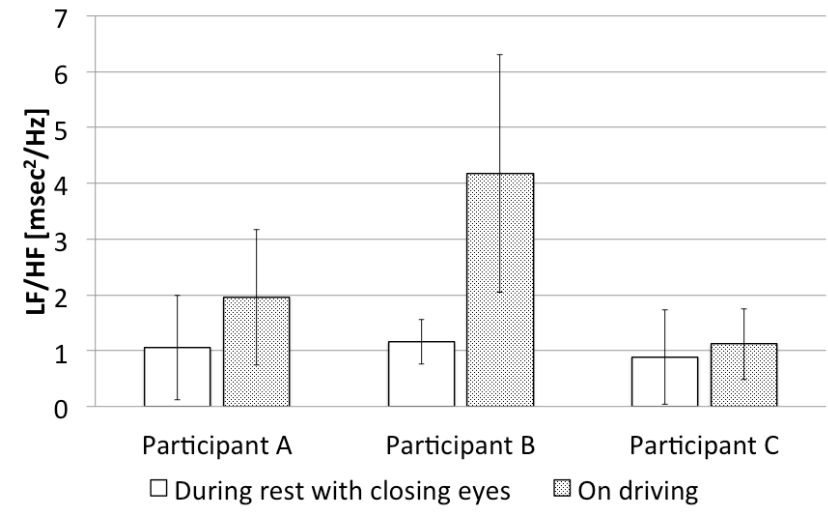

Fig. 1. LF/HF value of all participants when they were rest with closing eyes and on driving.

Table 1. LF/HF value of all participants when they were rest with closing eyes and on driving.

\begin{tabular}{lcc}
\hline & $\begin{array}{c}\text { During rest with closing } \\
\text { eyes }\end{array}$ & On driving \\
\hline \hline Participant A & $1.05 \pm 0.94$ & $1.95 \pm 1.21$ \\
Participant B & $1.16 \pm 0.40$ & $4.18 \pm 2.13$ \\
Participant C & $0.89 \pm 0.85$ & $1.12 \pm 0.64$ \\
\hline
\end{tabular}
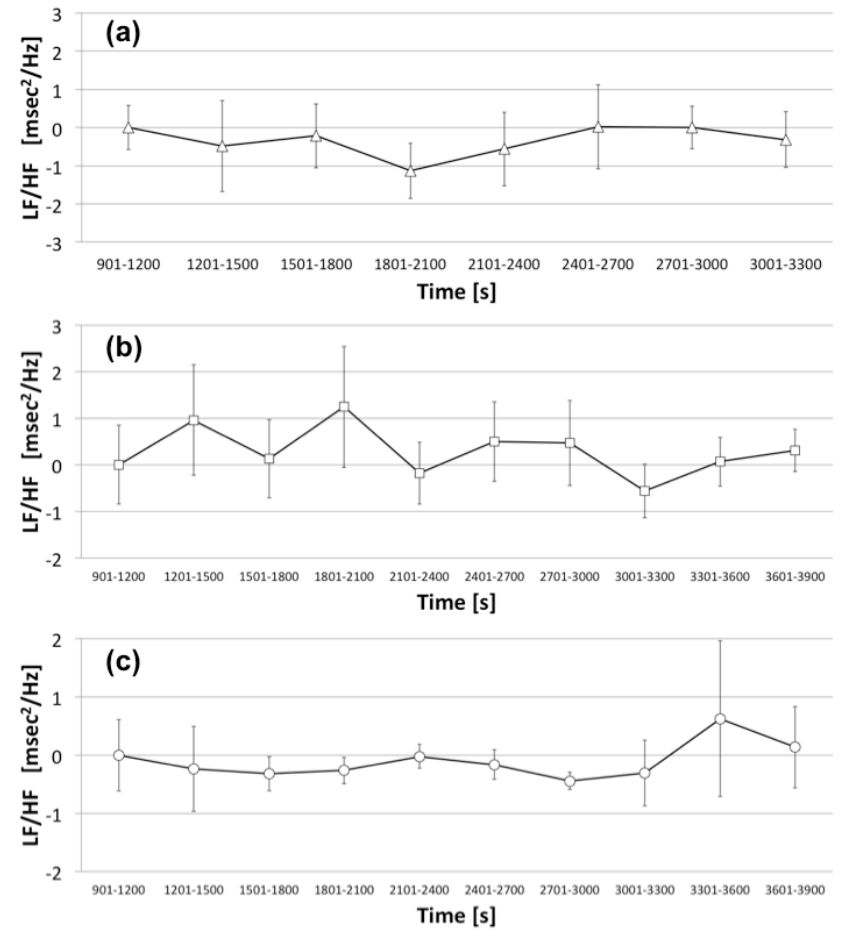

Fig. 2. Average LF/HF value of each 5 minutes from 15 minutes after start of driving. Participant B (b) and participant $\mathrm{C}$ (c) shows the tendency that the $\mathrm{LF} / \mathrm{HF}$ value get to increase with time. On the other hand, LF/HF value of participant A (a) of 15 minutes after star of driving is as the same as that at the end of driving. 
Table 2. Average LF/HF value of each 5 minutes from 15 minutes after start of driving.

\begin{tabular}{cccc}
\hline Seconds & Participant A & Participant B & Participant C \\
\hline \hline $901-1200$ & $0.00 \pm 0.58$ & $0.00 \pm 0.84$ & $0.00 \pm 0.61$ \\
$1201-1500$ & $-0.48 \pm 1.19$ & $0.96 \pm 1.18 * *$ & $-0.23 \pm 0.73$ \\
$1501-1800$ & $-0.22 \pm 0.83$ & $0.13 \pm 0.83$ & $-0.32 \pm 0.30$ \\
$1801-2100$ & $-1.13 \pm 0.72$ & $1.24 \pm 1.30 * *$ & $-0.26 \pm 0.23$ \\
$2101-2400$ & $-0.56 \pm 0.97$ & $-0.18 \pm 0.66$ & $-0.02 \pm 0.21$ \\
$2401-2700$ & $0.02 \pm 1.10$ & $0.50 \pm 0.86$ & $-0.16 \pm 0.26$ \\
$2701-3000$ & $0.00 \pm 0.56$ & $0.47 \pm 0.92$ & $-0.44 \pm 0.14$ \\
$3001-3300$ & $-0.31 \pm 0.73$ & $-0.56 \pm 0.57$ & $-0.31 \pm 0.56$ \\
$3301-3600$ & - & $0.07 \pm 0.53$ & $0.63 \pm 1.34 * *$ \\
$3601-3900$ & - & $0.31 \pm 0.45$ & $0.14 \pm 0.70$ \\
\hline
\end{tabular}

Data are mean \pm SD.

** $\mathrm{p}<0.01$ compared with $901-1200$ seconds.

because drivers were in "excessive state" and the LF/HF value was consequently high. From this reason, we analyzed LF/HF from 15 minutes after the start of driving.

From Fig. 2 and Table 2, z-transformed LF/HF value of participant $\mathrm{B}$ and $\mathrm{C}$ tends to increase with time though the $\mathrm{z}$-transformed LF/HF value has dispersion. From the result of "Jikaku-sho Shirabe" of participant A, the score of "yawning" and "drowsy" before experiment was already high, so it was clear that participant A was drowsy before experiment. Thus it is suggested that participant $\mathrm{A}$ had been already fatigue-like state and LF/HF value didn't increase while experiment.

\subsection{Trial Estimation of Fatigued State}

An easy method to estimate fatigued state is that the threshold of LF/HF value is determined and driver is considered as fatigued state when the LF/HF value excesses the determined threshold. For example, the threshold of $\mathrm{LF} / \mathrm{HF}$ value was determined as 1.5 and driver was considered as fatigued state when the $\mathrm{LF} / \mathrm{HF}$ value excesses 1.5. The result of all participants is shown as Fig. 3. From Fig. 3, it seems that participants' fatigued state was roughly detected and there is consistency based on introspective report on driving. However, this method doesn't consider the difference of each driver's characteristics, thus the method lacks of versatility. Thus, it is necessary to improve the detection algorithm.

\section{Conclusion}

From this experiment, it was found that the fatigue state can be detected by LF/HF. However, the total driving (a)

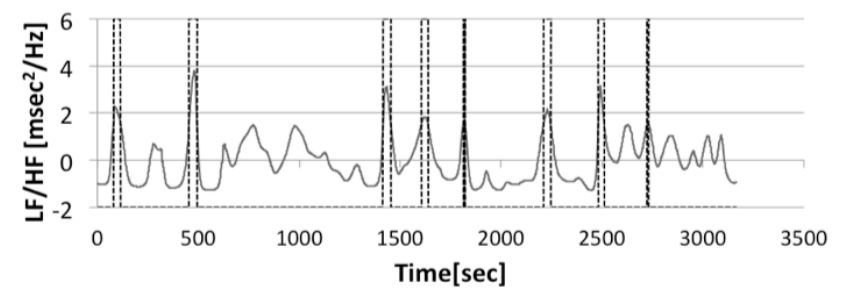

(b)

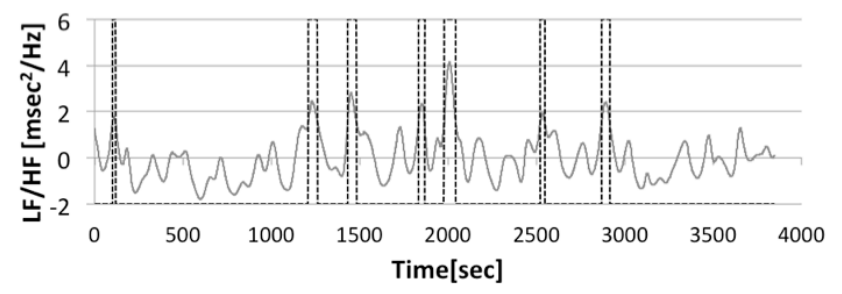

(c)

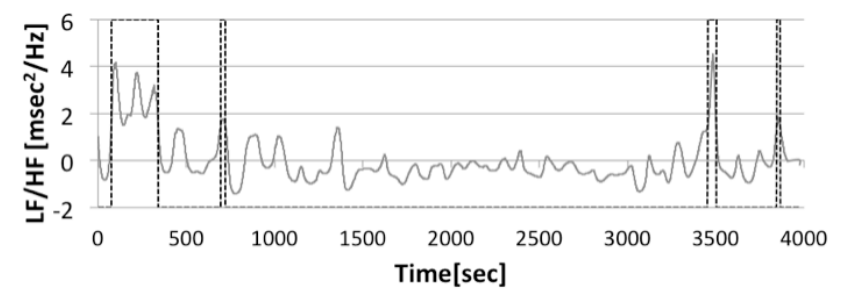

Fig.3. An Example of trial estimation of fatigued state. (a) is the estimation result of participant $A$, (b) is that of participant B and (c) is that of participant C. Driver (each participant) was considered as fatigued state when the LF/HF value excesses 1.5. From this figure, the result of detection is different from driver, and it is necessary to consider drivers' characteristics.

time of all participants were about 1 hour, and it is hard to say that they get to be fatigued state. In addition that, the participants were only 3 person. We will gather more participants and have participants drive more long time in order to get the LF/HF data by which driver's fatigued state is appeared more clearly.

\section{References}

(1) Expressway Dataroom Home Page. 4 June 2015 $<$ http://www.ne.jp/asahi/expressway/dataroom/>

(2) LACAN MENTAL SCIENCE Lab. Home Page. 26 January $2015<\mathrm{http}$ ://agency-inc.com/accident6/>

(3) Nihon Keizai Shinbun, Inc. Home Page. 16 January $2015<$ http:/www.nikkei.com/article/DGXNASDG030 1B T00C14A3CC0000/>

(4) L. R. Hartley, P. K. Arnold, G. Smythe and J. Hansen: "Indicators of fatigue in truck drivers", Appl. Ergonomics, 25, 3, 143-156, 1994. 
(5) Y. Kaseda, C. Jiang, K. Kurokawa, Y. Mimori and S. Nakamura: "Objective evaluation of fatigue by event-related potentials", J. Neurol. Sci., 158, 1, 96-100, 1998.

(6) S. Iwaki and N. Harada: "Mental fatigue measurement as application software on consumer devices Introducing reliable fatigue index to daily life -", Synthesiology, 4, 7, 220-227, 2014.

(7) S. Yamagishi and R. Tsuda: "Frequency Analysis of Mental Work Electrocardiogram", Proceedings of Industry-academic-government Technical Meeting in Kumamoto Pref, 2004.

(8) T. Arakawa, N. Matsuo and M. Kinoshita: "Trial Evaluation on Drivers' Unfocused Attention using Gaze Analysis", Review of Automotive Engineering, 27, 2, 357-359, 2006. 